\title{
Familiale Pflege: „echt unersetzlich...!?“
}

\section{Erste Online-Beratung für pflegende Jugendliche gestartet}

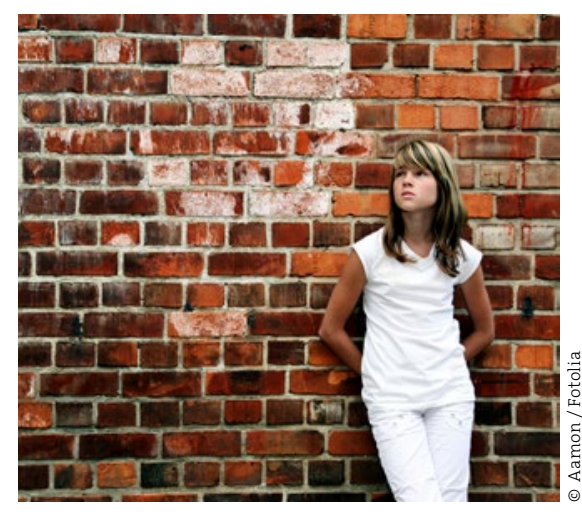

" Jugendliche, die ihre Familienangehörigen pflegen, werden in der Öffentlichkeit kaum wahrgenommen. Dabei sind es allein in Berlin schätzungsweise 30.000 , die ihre chronisch kranken oder behinderten Eltern, Großeltern oder Geschwister versorgen. Mit der Pflege tragen die jungen Menschen nicht nur eine hohe Verantwortung. Die Belastung durch die vielfältigen Aufgaben, die sie übernehmen, kann dazu führen, dass sie in ihrer persönlichen Entwicklung und auch hinsichtlich ihrer Bildungschancen beeinträchtigt werden.

Bundesweit einmalig bietet die neue Onlineberatung „echt unersetzlich...??“ unter dem Dach von „Pflege in Not“ Unterstützung und Begleitung für Jugendliche und junge Erwachsene mit Pflegeverantwortung an. Neben der Onlineinformation und der persönlichen und telefonischen Beratung gibt es bei der Onlineberatung krankheitsübergreifend und anonym Hilfestellungen für Betroffene.
Benjamin Salzmann („echt unersetzlich...!?“) erläutert: „Das Internet ist für Jugendliche bei solch schambesetzen Themen ein sehr guter Zugangsweg. Durch die Anonymität der Onlineberatung fühlen sich betroffene Jugendliche wohler, schwierige Themen wie Überlastung, Scham und Ängste anzusprechen. Auch bieten wir die Möglichkeit, jederzeit auch eine intensive, persönliche Beratung zu nutzen und wir geben ihnen die Möglichkeit, in Kontakt zu anderen betroffene Jugendlichen zu kommen."

Träger von „Pflege in Not“ ist das Diakonische Werk Berlin Stadtmitte e.V.

\section{www.echt-unersetzlich.de}

\section{Symposium Pflegemanagement der Charité}

\section{Fragen an Judith Heepe}

Am 25. November veranstaltet die Berliner Charité ihr erstes Pflegemanagement-

Symposium. Darüber sprachen wir mit der Pflegedirektorin Judith Heepe, unter deren Leitung das Symposium tagt.

Frau Heepe, warum veranstaltet die Charité ein Pflegemanagement-Symposium?

Heepe: Die Charité bietet für angehende Führungskräfte verschiedene Entwicklungsprogramme. Wir arbeiten mit einem Mentoringprogramm und bieten ein Traineeship für Managementinteressierte an. Diese neuen Karriereoptionen wollen wir gerne vorstellen und mit den Teilnehmern diskutieren.

Was zeichnet das Pflegemanagement-Symposium der Charité aus? Ist es offen für viele oder eher eine closed shop-Veranstaltung?

Heepe: Das Symposium ist definitiv keine closed shop Veranstaltung. Es soll ein Forum für einen interkollegialen Austausch bieten und Raum für kreative neue Entwicklungen eröffnen.
Der Schwerpunkt des Symposiums liegt auf der „Personalentwicklung" - warum?

Heepe: Führende Positionen zu bekleiden bedeutet, dass man Prozesse aktiv gestalten kann. Wir möchten daher Menschen für eine verantwortungsvolle Position begeistern. Die beträchtlichen Herausforderungen, die mit einer solchen Position verknüpft sind, als Chance zur Veränderung erleben zu können, ist unabdingbar mit einem Entwicklungsprozess verbunden.

Spannend finde ich auch den Aspekt „Frauen in Führungsrollen“. Wie erleben Sie diese Rolle und was werden Sie den Teilnehmern dazu mit auf den Weg geben?

Heepe: Natürlich konnten wir in unserer Gesellschaft bereits viel zur Gleichberechtigung der Frauen bei- tragen. Gleichwohl sind Frauen in leitenden

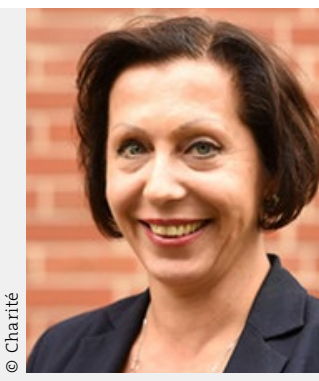
Judith Heepe, Pflegedirektorin der Charité in Berlin
Funktionen

terrepräsentiert. Daher wollen wirim Rahmen des Symposiums die möglichen Ursachen hierfür beleuchten und mögliche Lösungsansätze vorstellen.

Kann man sich noch anmelden oder sind die Plätze schon belegt? Heepe: Sehr positiv ist das große Interesse an der Veranstaltung. Noch gibt es wenige Plätze und wir freuen uns natürlich über weitere Anmeldungen (www.charite.de).

Das Interview führte Katja Kupfer-Geißler 